\title{
DIVERGÊNCIAS E CONVERGÊNCIAS ENTRE UM MODELO DE ASSISTÊNCIA DE ENFERMAGEM A PACIENTES DIABÉTICOS E A TEORIA DO DÉFICIT DE AUTOCUIDADO DE OREM
}

\author{
Marisa Ribeiro Bastos Peixoto*
}

PEIXOTO, M.R.B. Divergências e convergências entre um modelo de assistência de enfermagem a pacientes diabéticos e a teoria do déficit de autocuidado de OREM. Rev.Esc.Enf.USP, v. 30, n.1, p.1-13, abr. 1996.

São apresentados os pontos divergentes e convergentes entre um modelo de assistência a diabéticos e a teoria do Déficit de autocuidado de OREM. O modelo analisado pode ser considerado um programa de educação para pacientes diabéticos, diferindo nos aspectos conceituais e operacionais da teoria de OREM.

UNITERMOS: Autocuidado. Assistência a diabéticos.

\section{INTRODUÇÃO}

A assistência de enfermagem a pacientes com ênfase no autocuidado tem sido uma alternativa encontrada no sentido de, não só estimular o paciente a participar ativamente no seu tratamento, como também aumentar sua responsabilidade nos resultados da assistência.

Alguns trabalhos apontam as vantagens da aplicação do modelo de OREM na assistência a pacientes, tais como facilitar o planejamento de uma assistência de enfermagem ampla e efetiva a nível domiciliar e da equipe multidisciplinar (TITUS; PORTER, 1989). Da mesma forma, FOOTE et al. (1993) afirmam que a teoria de OREM fornece uma estrutura para uma assistência de enfermagem holística, nos aspectos relativos ao levantamento e interpretação dos dados.

\footnotetext{
* Enfermeira, Professor Assistente Mestre do Departamento de Enfermagem Básica da Escola de Enfermagem da Universidade Federal de Minas Gerais. Doutoranda do Programa de Expansão do Doutorado da Escola de Enfermagem da Universidade de São Paulo na Escola de Enfermagem da UFMG.
} 
Grande parte dos ambulatórios de assistência a pacientes diabéticos tem adotado modelos denominados de "autocuidado", onde a responsabilidade pela identificação de sinais e sintomas, controle da dieta, administração de insulina, bem como tomada de decisões a respeito do tratamento têm sido compartilhados entre pacientes e profissionais de saúde.

O termo autocuidado foi conceituado a partir da teoria de OREM. Segundo a autora, o autocuidado é a prática de atividades que as pessoas desempenham em seu proprio benefício, no sentido de manter benefício, no sentido de manter a vida, a saúde e o bem-estar (OREM, 1991).

Utilizando os pressupostos da teoria do autocuidado e da Teoria de Sistemas de Enfermagem, OREM (1991) descreveu a Teoria do Déficit de Autocuidado. Segundo essa teoria, o engajamento dos indivíduos nas práticas de autocuidado depende, em grande parte, de aspectos culturais, educacionais, relativos a habilidades e limitações pessoais, experiência de vida, estado de saúde e recursos disponíveis. A Teoria do Déficit de Autocuidado, segundo OREM(1991), estabelece a relação existente entre a capacidade de ação do indivíduo e a sua demanda para o autocuidado. Desta forma, o termo déficit se refere a uma relação entre as ações que os indivíduos deveriam desenvolver e aquelas que têm capacidade de desenvolver no sentido de manter a vida, a saúde e o bem-estar.

O enfermeiro, segundo a Teoria do Deficit de Autocuidado, é um indivíduo que possui habilidades e conhecimentos para identificar incapacidades às quais os seres humanos estão sujeitos. Essas incapacidades podem ser decorrentes do estado de saúde do indivíduo, impedindo-o de cuidar de si por certo período de tempo.

Assim, na Teoria do Déficit de Autocuidado, três conceitos são fundamentais: o conceito de agente de autocuidado, de demanda de autocuidado terapêutico e de déficit de autocuidado. Além desses conceitos, são importantes os aspectos relativos aos requisitos de autocuidado e os fatores condicionantes para o autocuidado.

Agente de autocuidado: é a capacidade dos indivíduos em desenvolver ação no sentido de regular o seu desenvolvimento e funcionamento; definir o que pode e o que deve ser feito e desenvolver as atividades selecionadas no sentido de atender as demandas de autocuidado. Quando existe incapacidade para o desempenho dessas ações (problemas de saúde, por exemplo), o enfermeiro ou elemento da enfermagem pode assumir por um certo período de tempo a situação de agente de autocuidado, ou seja, aquele elemento que possui características e habilidades específicas para desenvolver ações para o autocuidado de outros indivíduos.

Demanda de autocuidado terapêutico: são as medidas de autocuidado necessárias para atender os requisitos de autocuidado do tipo universal, de desenvolvimento ou de desvios de saúde, ou seja, é o conjunto 
de ações julgadas válidas e confiáveis no sentido de controlar fatores que afetam o desenvolvimento e regulação do corpo humano (OREM, 1991).

Segundo OREM (1991) as demandas de autocuidado terapêutico variam na sua composição, complexidade e estabilidade, de acordo com os requisitos de autocuidado que lhes deu origem.

Déficit de autocuidado: Na ótica de $\operatorname{OREM}(1991)$, o termo déficit de autocuidado não significa uma "desordem humana", podendo no entanto estar relacionada "com a figura de uma desordem funcional ou estrutural do homem". O Déficit de Autocuidado é identificado a partir da relação entre as demandas de autocuidado e a incapacidade de ação dos indivíduos para atendêlas.

Segundo OREM (1991), a enfermagem atua quando são identificados déficits para o autocuidado, estabelecendo com o paciente o seu plano de ação, envolvendo o estabelecimento da responsabilidade do enfermeiro, paciente e outros profissionais, no atendimento das demandas terapêuticas para o autocuidado.

ROURKE (1991) afirma que a negociação entre paciente e profissional sobre o estabelecimento do plano, é uma importante estratégia para o engajamento no autocuidado. $\mathrm{Na}$ visão do autor esta estratégia pode desencadear a auto-estima e a motivação do paciente para aderir ao autocuidado.

Requisitos de autocuidado: OREM (1991) define três tipos de requisitos de autocuidado:

- Requisitos universais: são necessários a todos os seres humanos no sentido de "manter a integridade da estrutura e funcionamento do corpo humano e bem-estar geral". Estão associados aos processos vitais ou necessidades humanas básicas.

- Requisitos de desenvolvimento: referem-se aos vários estágios do ciclo de vida do homem, relacionando-se com o processo de desenvolvimento do homem e com aqueles fatores que os influencia.

- Requisitos de desvio de saúde: estão relacionados a problemas de natureza funcional, genética, bem como a diagnóstico médicos e medidas terapêuticas.

Fatores condicionantes para o autocuidado: são "fatores internos ou externos ao indivíduo que afetam sua capacidade de engajar no autocuidado", ou seja, idade, sexo, estado de desenvolvimento, estado de saúde; fatores socioculturais, familiares e ambientais; aspectos relativos ao sistema de assistência á saúde, padrão de vida e disponibilidade de recursos. (OREM, 1991)

A teoria acima descrita, ou alguns de seus pressupostos básicos, tem sido utilizada na definição de modelos assistenciais de enfermagem, sem 
haver, no entanto, uma correspondência entre este e o modelo descrito por OREM(1991).

Diante do exposto, buscamos analisar um modelo de assistência de enfermagem a pacientes diabéticos a nível ambulatorial, tendo como referência a Teoria do Déficit de Autocuidado de OREM (1991).

\section{OBJETIVO}

Identificar os pontos convergentes e divergentes entre o modelo de assistência de enfermagem a pacientes diabéticos, adotado no ambulatório em estudo, e a Teoria do Déficit de Autocuidado de OREM.

\section{METODOLOGIA}

A proposta de identificar os pontos divergentes e convergentes entre o modelo de assistência de enfermagem ao diabético, utilizado pela instituição estudada, e a Teoria do Déficit de Autocuidado, foi operacionalizada através da análise dos seguintes aspectos da teoria de OREM (1991):

a) Levantamento dos fatores condicionantes para o autocuidado:

-idade

- sexo

- estágio de desenvolvimento

- estado de saúde

- aspectos socioculturais

- fatores familiares

- padrão de vida

- Fatores ambientais.

b) Identificação das demandas terapêuticas para o autocuidado segundo os requisitos universais, de desenvolvimento e de desvios de saúde.

c) Levantamento das capacidades e habilidades do paciente para atender as demandas de autocuidado terapêutico.

d) Identificação de déficits de autocuidado.

e) Estabelecimento do sistema de enfermagem:

- planejamento da assistência

- participação do paciente.

f) Avaliação. 
Desta forma, buscamos identificar no modelo de assistência de enfermagem adotado na instituição, os itens acima citados, considerados neste estudo aspectos fundamentais da Teoria do Déficit de Autocuidado.

\subsection{Campo de Pesquisa e Participantes}

O Estudo foi realizado numa instituição de assistência a pacientes diabéticos em nível ambulatorial.

Participaram da pesquisa: uma enfermeira do setor e alunos de pósgraduação de Enfermagem que desenvolvem atividades naquele setor.

\subsection{Coleta e Análise dos Dados}

Os dados foram coletados por meio de entrevistas não estruturadas, observações e análise de documentos do setor.

As entrevistas não estruturadas foram realizadas com uma enfermeira e alunos de enfermagem com o objetivo de caracterizar o modelo de assistência adotado no setor.

Para cada atividade observada foi utilizado um impresso (anexo) onde foram analisados os aspectos da teoria de OREM considerados neste estudo. De acordo com cada atividade, era checada a realização do levantamento dos dados ou a utilização dos mesmos no desenvolvimento da atividade.

Foram observados: 4 primeiras consultas de enfermagem, 4 consultas subsequentes de enfermagem, 2 grupos operativos, 3 aplicações de insulina pela enfermagem e 1 auto-aplicação.

Foi realizada, também, análise de documentos utilizados no setor, tais como formulários, módulos de sensibilização, entre outros, com o objetivo de identificar aspectos considerados importantes do modelo em análise, bem como complementar as informações obtidas pelas outras técnicas de coleta de dados.

\section{CARACTERIZAÇÃO INSTITUCIONAL E DO MODELO DE ASSISTÊNCIA}

O estudo foi realizado em um ambulatório público de endocrinologia, caracterizado pelos profissionais do setor como "um serviço de orientação para o autocuidado", ou "serviço de educação para o autocuidado".

A planta física da instituição abrange 5 consultórios médicos, um consultório de enfermagem e um salão destinado ao desenvolvimento de práticas educativas, funcionando no período da manhã e da tarde.

$\mathrm{O}$ ambulatório atende a pacientes com afeç̧ões do sistema end6crino, sendo a maioria constituída por pacientes diabéticos, contando com médicos, 
enfermeiras, técnicas de enfermagem, secretária e funcionária administrativa.

O setor é o campo de estágio para o curso de graduação em Nutrição, graduação e pós-graduação de Enfermagem e Residência Médica.

E importante ressaltar que a equipe de enfermagem do setor, bem como os alunos de graduação e pós-graduação em enfermagem, desenvolvem um módulo de sensibilização (MINAS GERAIS, 1990), onde são abordados os aspectos epidemiologicos, fisiopatológicos e propedêuticos da Diabetes Mellitus.

O modelo de assistência de enfermagem adotado na instituição pode ser representado pelo diagrama abaixo:

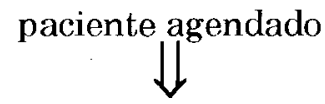

curso de introdução<smiles>[C]#C</smiles>

formação de grupos operativos<smiles>[C]1=CC=C1</smiles>

$1^{a}$ consulta de enfermagem (método clínico)<smiles>C=CC</smiles>

consultas subsequentes<smiles>[AlH2]</smiles>

grupos operativos

Após o agendamento do paciente no serviço de endocrinologia, o paciente participa de uma programação de aulas teóricas e práticas abrangendo os seguintes temas:

- conceito de Diabetes Mellitus

- fisiop atologia

- sinais e sintomas de hipo e hiperglicemia (aguda e crônica)

- dieta (grupos alimentares e tipos de alimentos)

- atividades físicas e cuidados com o corpo

- complicações

- medicação (insulinoterapia).

Estes temas são desenvolvidos através de 5 a 6 aulas, sendo a última voltada para a aprendizagem da auto-aplicação da insulina, contando com a participação de um farmacêutico. 
Ao término das aulas, é esclarecido aos pacientes que deverão retornar ao serviço mediante uma convocação pelo correio, onde constará a data e o horário de seu retorno.

Após a participação nas aulas, são formados grupos operativos de 12 pacientes, distribuídos pela enfermeira de acordo com a faixa etária e o tipo de diabetes.

No dia do primeiro retorno do paciente é realizada a primeira consulta com o enfermeiro ou aluno de enfermagem com utilização do método clínico. Existe um roteiro de avaliação do estado de saúde do cliente diabético para orientação da anamnese e exame físico. No dia da primeira consulta é também preenchido um impresso constando dados pessoais do cliente, tipo de diabetes, tratamento, identificação dos fatores de risco e complicações.

Os grupos operativos têm início em datas e horários marcados pelo serviço, sendo o paciente convocado, novamente, por carta. Neste dia, quando o paciente chega ao setor, é feita a glicemia que é anotada no quadro-negro da sala. Caso seja necessário, será administrada insulina e oferecido um lanche. Em todos os dias em que participam de grupos operativos, os pacientes têm consultas de enfermagem. Nessas consultas subsequentes não é feita a avaliação do estado de saúde do cliente com utilização do método clínico de forma integral. A enfermeira ou aluna preenche um impresso que consta apenas dos seguintes dados: data, número do grupo, nome, nome do médico, outros profissionais, sinais e sintomas atuais, complicações, medicação, peso, pressão arterial, centonúria, glicosúria, glicemia e estabelecimento das condutas de enfermagem.

Após a consulta, iniciam-se as atividades dos grupos operativos que são desenvolvidos através de discussões e/ou jogos, denominados "práticas educativas".

Ao final, os pacientes sugerem os temas que deverão ser abordados nas reuniões subsequentes.

\section{CONVERGÊNCIAS E DIVERGÊNCIAS ENTRE O MODELO DE ASSISTÊNCIA E A TEORIA DE OREM}

No modelo de assistência de enfermagem estudado é feito o levantamento dos requisitos de autocuidado, bem como dos fatores condicionantes, ou seja, dos fatores que afetam a capacidade do indivíduo para atender as demandas de autocuidado terapêutico. Esses dados são obtidos através da entrevista feita na primeira consulta e registrados no impresso próprio, sendo enfatizados os fatores de natureza descritiva, tais como: idade, sexo, ocupação, fatores socioculturais e os relativos ao estado físico e psicológico. 
No entanto, estes dados não são utilizados no sentido de identificar seus efeitos no atendimento das demandas de autocuidado terapêutico.

Segundo OREM (1991), a enfermeira deve fazer uma abordagem objetiva no sentido de investigar os efeitos desses fatores no atendimento das demandas de autocuidado terapêutico dos seus pacientes. Da mesma forma, como parte desta investigação, a enfermeira deve reconhecer como importantes as informações subjetivas do paciente a respeito de suas experiências pessoais no atendimento dos requisitos.(OREM, 1991).

Alguns desses fatores são estáveis, mas outros estão em contínua mudança. Sendo assim torna-se necessário que o enfermeiro obtenha informações relativas aos fatores condicionantes, freqüentemente, interrelacionando-os (OREM, 1991).

O modelo analisado, embora abrangendo aspectos individuais da assistência, adota uma abordagem coletiva no que se refere a demandas de autocuidado terapêutico. Da mesma forma, utiliza referências padronizadas para as capacidades individuais e déficit para o autocuidado, tais como o nível de conhecimento sobre a doença e tratamento, motivação para aderência às práticas de autocuidado e habilidades para auto-aplicação de insulina.

Os jogos operativos são desenvolvidos entre pacientes com diversas faixas etárias, diferentes níveis de conhecimento e motivação. Num dos jogos operativos observados, um paciente idoso, com deficiência auditiva grave, assistia a uma discussão sobre "cuidados com os pés", sem ouvir ou entender o que se passava à sua volta. Da mesma forma, um paciente permaneceu por 5 minutos com a cabeça recostada sobre a mesa, sem participar da discussão.

A identificação das demandas de autocuidado terapêutico, na perspectiva de OREM (1991) e feita através do levantamento dos requisitos de autocuidados de natureza universal, desenvolvimental e de desvios de saúde, e, a partir daí, da identificação das condições humanas ou ambientais que possibilitam, impedem ou dificultam o seu atendimento. São identificados déficits quando as ações do paciente são consideradas insuficientes ou inadequadas para atender as suas demandas de autocuidado terapêutico ou se existir uma incapacidade potencial para satisfazê-las. Desta forma, o levantamento de demandas e déficits de autocuidado, bem como o estabelecimento do sistema de enfermagem e plano de ação, é um processo único e individualizado, compartilhado entre profissional e cliente.

O setor des envolve um programa de educação que difere da concepção de educação para o autocuidado de OREM (1991) em alguns aspectos básicos.

Analisando os aspectos conceituais da Teoria do Autocuidado, ROURKE (1991) afirma que, de acordo com OREM (1991), é de fundamental importância que o indivíduo seja reconhecido como aquele que tem o direito de exercer o controle sobre si e sobre sua assistência, numa relação cooperativa com os profissionais de saúde. 
Sob a perspectiva de DUMAS (1992), o indivíduo desenvolve ações específicas e deliberadas para manter sua vida e bem-estar.

Assim, é fundamental que, ao elaborar o plano de assistência, seja estabelecidos os objetivos e prioridades de forma compartilhada, onde os valores e crenças do indivíduo, nível de conhecimento, habilidades e motivação individual sejam considerados. Na educação para o autocuidado o paciente deve participar das decisões sobre as necessidades educativas, conteúdo, objetivos a alcançar e métodos utilizados (LEVIN, 1978).

No modelo analisado a avaliação se dá de forma assistemática, o que difere significativamente do modelo de OREM onde a fase de avaliação é muito importante. Segundo OREM (1991), esta fase compreende não s6 o acompanhamento dos procedimentos prescritos no plano de assistência, como também a avaliação da pertinência das metas estabelecidas em relação as condições pessoais e ambientais do paciente. Desta forma, é importante avaliar se os objetivos estabelecidos no plano estão sendo alcançados, bem como os resultados relativos ao engajamento do paciente às práticas de autocuidado.

De acordo com JOSEPH (1982), a avaliação do sistema de enfermagem deve tomar como critério para desempenho, os objetivos do paciente, observando se o resultado esperado foi atingido, a resposta do paciente às ações de enfermagem, as atividades de enfermagem, bem como avaliar as possíveis mudanças no sistema de enfermagem.

\section{CONSIDERAÇÕES CONCLUSIVAS}

O modelo de assistência de enfermagem analisado pode ser considerado um programa de educação para pacientes diabéticos que, embora seja denominado de "educação para o au tocuidado", difere nos aspectos conceituais e operacionais da teoria descrita por (OREM,1991).

Frente aos aspectos da Teoria do Déficit de Autocuidado, considerados neste estudo, podemos constatar divergências e convergências entre a teoria de OREM (1991) e o modelo de assistência analisado.

As principais divergências se referem a ausência de uma abordagem individualizada e sistemática no que se relaciona à identificação de demandas de autocuidado terapêutico, capacidades para o autocuidado e déficits.

No modelo analisado, os déficits para o autocuidado relativos a necessidade de conhecimento sobre a doença e tratamento, habilidades e motivação são considerados de forma coletiva e, embora o programa seja desenvolvido no sentido de dar independência e informação ao cliente, os objetivos e prioridades são, basicamente, estabelecidos pelos profissionais, o que difere, sob o ponto de vista conceitual e operacional, da teoria de (OREM, 1991) 
O indivíduo é considerado dentro de um contexto mais amplo, sujeito a condições ambientais, sociais e humanas que facilitam ou limitam sua capacidade para se engajar no au tocuidado. Sob essa 6tica, o modelo encontra consonância com os pressupostos básicos da teoria de (OREM,1991). No entanto, estes dados não são utilizados para o cálculo de demandas terapêuticas, capacidades e déficits.

Concluindo, concordamos com LEVIN (1978), quando afirma que não se pode denominar os programas de educação como sinônimos de programas de educação para o autocuidado, visto que têm objetivos, estratégias e pressupostos teóricos diversos. Na visão do autor, os conteúdos repressivos da educação formal são incompatíveis com os valores do autocuidado, assim como o conceito de aderência que não está, necessariamente, ligado ao interesse do cliente (LEVIN, 1978).

Segundo LANGE et al. (1989),

"a implementação do modelo de atenção
à saúde com ênfase no autocuidado
implica introduzir algumas mudanças na
forma em que tradicionalmente são
atendidas as pessoas no serviços de
saúde."

A atenção à saúde, dentro da concepção filosófica do autocuidado, remete a mudanças no que se refere ao funcionamento dos serviços, exigindo uma flexibilização das normas institucionais; do papel dos profissionais, exigindo um repensar de sua prática; e do papel do usuário, na tomada de decisão, controle e condução da assistência.

Resta ressaltar que a assistência a pacientes diabéticos, pautada no autocuidado, tem mostrado bons resultados no que se refere a aderência do paciente às medidas terapêuticas prescritas, bem como na conscientização do paciente com relação aos efeitos da doença e responsabilidade pelo seu controle. No estudo com jovens diabéticos feito por FREY, FOX (1990), foi identificado que existe uma relação significativa entre engajamento nas práticas de autocuidado e o controle metabólico e autopercepção do estado de saúde. Os autores concluem que o desenvolvimento das práticas de autocuidado, como foram conceitualizados por OREM(1991), é de fundamental importância para o controle de diabetes. 
PEIXOTO, M.R.B. Divergences and convergences between nursing assistance model for diabetic patients and the OREM's self-care deficit theory. Rev.Esc.Enf.USP, v.30, n.1, p.1-13,Apr. 1996.

The divergences and convergences between the Nursing Assistance model for diabetic patients and the OREM's Self-care Deficit theory are presented. The model studied may be considered an educational program for diabetic patients, differing on the conceptual aspects of OREM's theory.

UNITERMS: Self-care. Assistance to diabetes.

\section{REFERÊNCIAS BIBLIOGRÁFICAS}

DUMAS, L. La démarche de soins selon OREM. Can Nurse v.88, n.6, p.36-9, 1992.

FOOTE, A. et al. OREM's theory used as a guide for the nursing care of an eight-year-old child with Leukemia. J. Pediatr Oncol Nurs, v. 10, n.1, p.26-32, 1993.

FREY, M.; FOX. M. A. Assessing and teaching self-care to youths with diabetes Mellitus. Pediatr Nurs, v. 16, n.6, p. 597-9, 1990.

JOSEPH, L. S. Self-care and the nursing process. Nurs Clin North Am. v. 15, n. 1, p. 131-43, 1982.

LANGE, I. et al. Atención de salud con enfasis en autocuidado. IN: CAMPOS, C. et al. Enfermeria para la atención primaria. Chile, Projecto LEAP/Kellog, 1992. p. 10-5.

LEVIN, L . Patient education and self-care: How do they differ? Nurs Outlook, v. 3, n. 26, p. $170-5,1978$.

MINAS GERAIS, (Estado) Prefeitura Municipal. Secretaria Municipal de Saúde. Diabetes Mellitus: módulo de sensibilização. Belo Horizonte, 1990.

OREM, D. E. Nursing: Concepts of practice. 4. ed. Saint. Louis, Mosby, 1991

ROURKE, A. M. Self-care: chore or challenge? J Adv Nurs.v.16, n.2, p.233-41, 1991.

TITUS, S.; PORTER, P. OREM's theory applied to pediatric residential treatment. Pediatr Nurs. v. 15, n.5, p.465-8, 1989. 


\section{ANEXO}

Data

Atividade Observada

Aspectos da Teoria de OREM observados:

1 Fatores condicionantes

idade

sexo

estágio de desenvolvimento

estado de saúde

aspectos socioculturais

fatores familiares

padrão de vida

fatores ambientais

Observações:

2 Demandas terapêuticas para o autocuidado

- requisitos universais

- requisitos de desenvolvimento

- requisitos de desvio de saúde

Observações:

$-$

3 Capacidades e habilidades do paciente para atender as demandas de autocuidado terapêutico.

4 Déficits de autocuidado 
5 Sistema de enfermagem

- planejamento da assistência

- participação do paciente

- Observações:

6 Avaliação 